\title{
PAPER
}

\section{Standard magnetic resonance imaging is inadequate for patients with refractory focal epilepsy}

\author{
J von Oertzen, H Urbach, S Jungbluth, M Kurthen, M Reuber, G Fernández, C E Elger
}

See Editorial Commentary pages 612-13

J Neurol Neurosurg Psychiatry 2002;73:643-647

See end of article for authors' affiliations

.....................

Correspondence to:

Dr J von Oertzen

Department of

Epileptology, University of

Bonn, Sigmund-Freud-Str

25,53105 Bonn

Germany;

joachim-von.oertzen@

ukb.uni-bonn.de

Received 22 March 2002

In revised form 26 July

2002

Accepted 2 August 2002

\begin{abstract}
Objectives: Patients with intractable epilepsy may benefit from epilepsy surgery especially if they have a radiologically demonstrable cerebral lesion. Dedicated magnetic resonance imaging (MRI) protocols as performed at epilepsy surgery centres can detect epileptogenic abnormalities with great sensitivity and specificity. However, many patients with epilepsy are investigated with standard MRI sequences by radiologist outside epilepsy centres ("non-experts"). This study was undertaken to compare standard MRI and epilepsy specific MRI findings in patients with focal epilepsy.

Methods: Comparison of results of standard MRI reported by "non-expert" radiologists, standard MRI evaluated by epilepsy "expert" radiologists, and epilepsy specific MRI read by "expert" radiologists in 123 consecutive patients undergoing epilepsy surgery evaluation between 1996 and 1999. Validation of radiological findings by correlation with postoperative histological examination.

Results: Sensitivity of "non-expert" reports of standard MRI reports for focal lesions was 39\%, of "expert" reports of standard MRI 50\%, and of epilepsy dedicated MRI 91\%. Dedicated MRI showed focal lesions in $85 \%$ of patients with "non-lesional" standard MRI. The technical quality of standard MRI improved during the study period, but "non-expert" reporting did not. In particular, hippocampal sclerosis was missed in $86 \%$ of cases. Neuropathological diagnoses $(n=90)$ were predicted correctly in $22 \%$ of "non-expert" standard MRI reports but by $89 \%$ of dedicated MRI reports.

Conclusions: Standard MRI failed to detect $57 \%$ of focal epileptogenic lesions. Patients without MRI lesion are less likely to be considered candidates for epilepsy surgery. Patients with refractory epilepsy should be referred to an MRI unit with epileptological experience at an early point.
\end{abstract}

$\mathrm{T}$ he prevalence of epilepsy is $0.5 \%-1 \% .^{1}$ About $75 \%$ of patients with epilepsy can be treated satisfactorily with antiepileptic drugs. However, treated success depends on epilepsy syndrome and seizure types. For example, antiepileptic drugs fail to control seizures in up to $75 \%$ of localisation related epilepsies. ${ }^{2-5}$ For medically intractable epilepsy patients, surgical treatment is the major therapeutic alternative. About $70 \%$ of patients who undergo epilepsy surgery become seizure free. The success rate may be even higher in patients with medial temporal lobe epilepsy. ${ }^{67}$

As a result of the constant improvement of image quality, magnetic resonance imaging (MRI) has become one of the most important tools in the selection of patients for epilepsy surgery. ${ }^{8}$ Congruity of a radiologically demonstrable lesion, ictal epileptic discharges in the EEG, and seizure semiology may permit epilepsy surgery without further invasive diagnostic procedures. In patients in whom scalp EEG recordings are insufficient, MRI abnormalities may generate a hypothesis for intracranial electrode implantation. ${ }^{6}$

Often, MRI is performed repeatedly during the course of a seizure disorder. It may be undertaken to aid the initial diagnostic process and repeated in patients proving refractory to treatment. MRI protocols with high specificity and sensitivity for epileptogenic lesions have been developed and tested in epilepsy centres. These protocols involve thin slice thickness, high resolution, and, in temporal lobe epilepsy patients, orientation perpendicular or parallel to the longitudinal axis of the hippocampal body. However, many patients with epilepsy continue to be investigated using standard MRI "head" sequences. A previous, smaller study comparing standard MRI protocols with a protocol dedicated to the investigation of epilepsy patients between 1993 and 1996 focused exclusively on temporal lobe epilepsy patients. ${ }^{10}$ In our study, we re-evaluate the sensitivity and specificity of standard versus dedicated MRI in an unselected and larger sample of patients. MRI findings were validated by histopathological results after epilepsy surgery.

\section{METHODS}

The records of 536 consecutive patients undergoing presurgical evaluation between January 1996 and June 1999 were reviewed. We included all patients whose records contained all of the following documents: (1) report of standard MRI by the performing (neuro-)radiologist; (2) report of "expert" reassessment of standard MRI; and (3) report of MRI using a dedicated epilepsy protocol. Patients were excluded because at least one of the MRI reports was missing (usually because MRI had not been performed outside our centre or standard MRI was not available for reporting by our "expert" neuroradiologists). Patients with rapidly changing epileptogenic lesions (for example, high grade tumours) were also excluded.

Standard MRIs were undertaken and reported by radiologists not attached to epilepsy centres ("non-experts"). Standard MRI was reported by general radiologists in $47 \%$, and by neuroradiologists in $25 \%$ of cases. The qualification of the reporting radiologist was not specified in $28 \%$ of standard MRI reports. Reassessment was performed by one of five neuroradiologists with more than three years epileptological experience based in the radiology department of our epilepsy centre ("experts"). MR images were usually re-reported at the time of the patient's first visit to our outpatient department. The "expert" neuroradiologists were informed of the seizure semiology at the time of re-reporting of standard MRI. MRI using our dedicated epilepsy protocol was performed during

Abbreviations: $M R I$, magnetic resonance imaging; $\mathrm{HS}$, hippocampal sclerosis 


\begin{tabular}{l} 
Table 1 Classification of MRI and histological \\
diagnoses as used in this study. \\
\hline Normal or no focal abnormality \\
Insufficient quality of standard MRI \\
Hippocampal sclerosis \\
Migration disorders \\
cortical dysplasia \\
heterotopia \\
phacomatosis \\
Tumour \\
dysembryoplastic neuroepithelial tumour (DNT) \\
ganglioglioma \\
low grade astrocytoma (WHO I-II) \\
Vascular malformation \\
AV malformation \\
cavernoma \\
Other focal lesions \\
gliosis \\
post-encephalitis \\
cystic lesion \\
post-infarction \\
post-contusion \\
\hline
\end{tabular}

inpatient presurgical evaluation and assessed by the same group of "experts". The "expert" neuroradiologists were informed of seizure semiology to aid MRI orientation but they were blinded to other patient data. In particular, "experts" reporting epilepsy dedicated MRI were unaware of previous "expert" or "non-expert" reports of standard MRI.

The inclusion criteria were met by 123 patients. Imaging results could be correlated with neuropathological findings in 90 of these 123 patients who went on to have epilepsy surgery. Most of the 33 patients who did not undergo an operation were considered poor surgical candidates (because of bilateral seizure onset zones, seizure onset zone in eloquent brain areas, or missing hypothesis for placement of intracranial electrodes). A few patients decided against an operation.

Findings were classified into seven categories according to pathological entity (table 1). Standard MRI were considered technically inadequate if patients were scanned in less than three different orientations, or if image quality was unsatisfactory (for example, because of artefacts, low contrast, or low resolution)

The interval between MRI scans using standard and dedicated epilepsy protocols was quoted when exact examination dates were available $(\mathrm{n}=90)$. Patients were divided into an "abnormal" $(\mathrm{n}=51)$ and "normal" $(\mathrm{n}=39)$ group according to "non-expert" standard MRI reports, and a Wilcoxon signed rank test was calculated (SPSS 10.0, SPSS, Chicago, IL). As the groups differed in size, 12 patients were randomly excluded from the lesional group before the test was performed. The test was repeated five times with different exclusions to ensure that outcome was not biased by exclusions.

MRI according to our dedicated epilepsy protocol was performed on a 1.5 T scanner (Gyroscan ACS-II Philips Medical Systems, Best, Netherlands). Field of view was $220 \times 220$ $\mathrm{mm}$ and matrix was $256 \times 256$. The protocol included: sagittal Tl weighted spin echo sequence $(5.0 \mathrm{~mm}$ slice thickness, 0.5 mm interslice gap, TR $650 \mathrm{~ms}$, TE $16 \mathrm{~ms}$ ), axial T2 weighted TSE sequence $(5.0 / 1.0 / 2876 / 120)$, coronal T2 weighted TSE sequence $(2.0 / 0.3 / 3719 / 120)$, coronal Tl weighted inversion recovery sequence (6.0/1.2/2300/40/TI $470 \mathrm{~ms})$, and axial FLAIR sequence (5.0/1.0/6000/120/1900). In patients with temporal lobe epilepsy, sequences were angulated perpendicularly or parallel to the longitudinal axis of the hippocampal body. The dedicated epilepsy protocol used in this study did not include any quantitative MRI (hippocampal volumetry or $\mathrm{T} 2$ relaxometry).

\section{RESULTS}

\section{All patients $(n=123)$}

"Non-experts" reported $61 \%(\mathrm{n}=75)$ of standard MR scans as normal or as showing no focal abnormality (fig 1A, 2, 3A, B). Overall, focal lesions were reported in $39 \%(n=48)$ of cases ( fig 4A). Hippocampal sclerosis (HS) was detected in $7 \%(n=8)$ of patients (fig lA). In the "non-expert" group there were no significant discrepancies between results of general radiologists or neuroradiologists.

"Expert" reassessment of the same standard MRI classified $28 \%(n=34)$ of scans as technically inadequate (although abnormalities were suspected in 8 of 34 patients). "Experts" considered $22 \%(n=27)$ of standard MRI scans as normal or to show non-focal abnormalities only (fig 4A). "Experts" found focal lesions in $50 \%(n=62)$ of standard MRI scans (fig 2$)$, HS was diagnosed in $18 \%(n=22)$ of cases.

Using the dedicated epilepsy MRI protocol, the same group of "experts" described focal abnormalities in 91\% $(n=112)$ of scans (fig 1B, 3C, D). HS was detected in $45 \%(n=55)$ of cases (fig $1 \mathrm{Bl}$ ). For details of results see figure $4 \mathrm{~A}$.

With respect to localisation, "non-expert" standard MRI reports described mesial temporal lobe abnormalities in 9\% $(\mathrm{n}=11)$ and neocortical lesions in $31 \%(\mathrm{n}=38)$ of patients. "Expert" reassessment of standard MRI revealed mesial temporal abnormalities and neocortical lesions in $28 \%(n=34)$ and $23 \%(n=28)$, and dedicated MRI in $50 \%(n=62)$ and $41 \%$ $(\mathrm{n}=50)$ of cases.
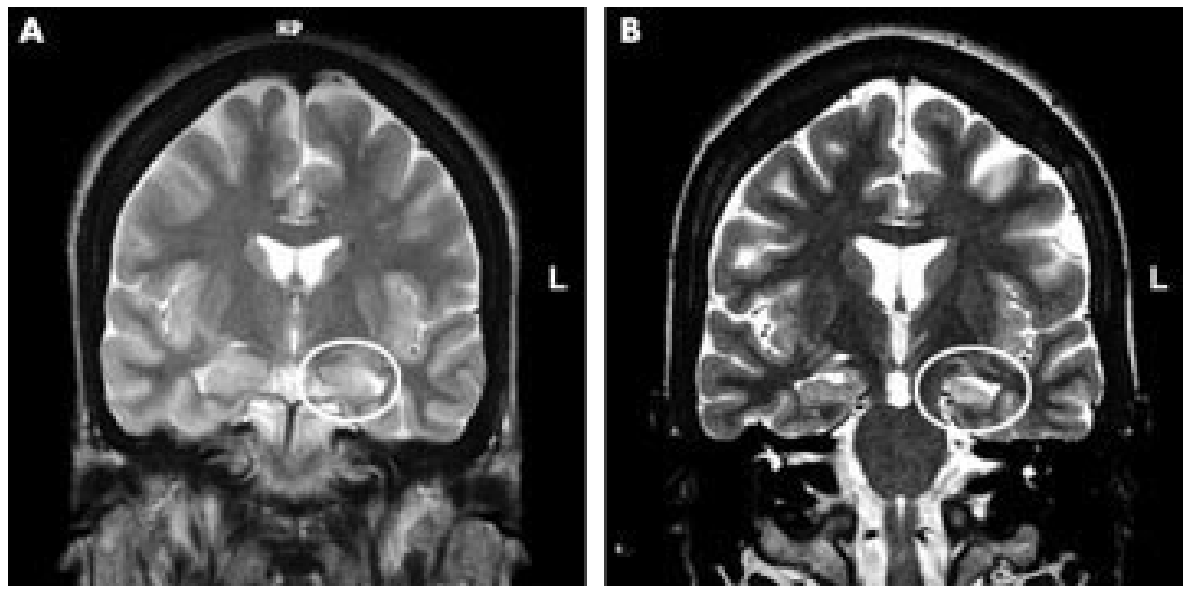

Figure 1 Insufficient quality of standard MRI: T2 weighted standard MRI (A) and epilepsy dedicated MRI (B) angulated coronally, orthogonally to the AC-PC plane (A), and orthogonally to the longitudinal axis of the hippocampus (B) (see fig 3). Standard MRI (A) shows no signal abnormalities or atrophy of hippocampus. 

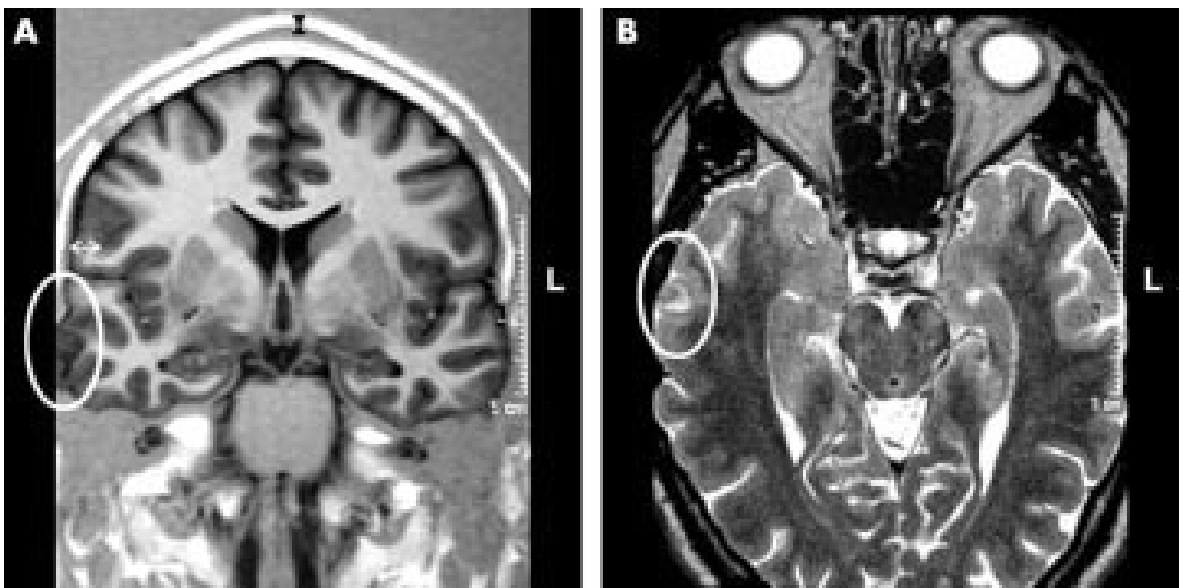

Figure 2 Failure of "non-expert" assessment: coronal T1 weighted inversion recovery (A) and axial T2 weighted fast spin echo sequences (B) of standard MRI reported as normal. The right temporo-lateral ganglioglioma on the margin of the image was not seen.

\section{Epilepsy surgery patients $(n=90)$}

The likelihood of correct diagnosis of "non-expert" standard MRI findings, reassessed standard, and dedicated epilepsy MRI protocols is given in Fig 4B. The overall specificity for focal lesions of standard MRI findings was $22 \%(n=20)$, if re-assessed by "experts" $40 \%(n=36)$, and of dedicated MRI $89 \%(\mathrm{n}=80)$. Comparison with histopathological reports revealed that dedicated MRI was best at predicting HS and vascular malformations, whereas the identification of migration disorders was more difficult (fig 4B).

\section{Non-operated patients $(n=33)$}

Standard MRI of non-operated patients failed to detect any focal lesion in $70 \%(n=23)$, with "expert" reassessment in $58 \%(n=19)$, and dedicated MRI in $40 \%(n=13)$ of patients.
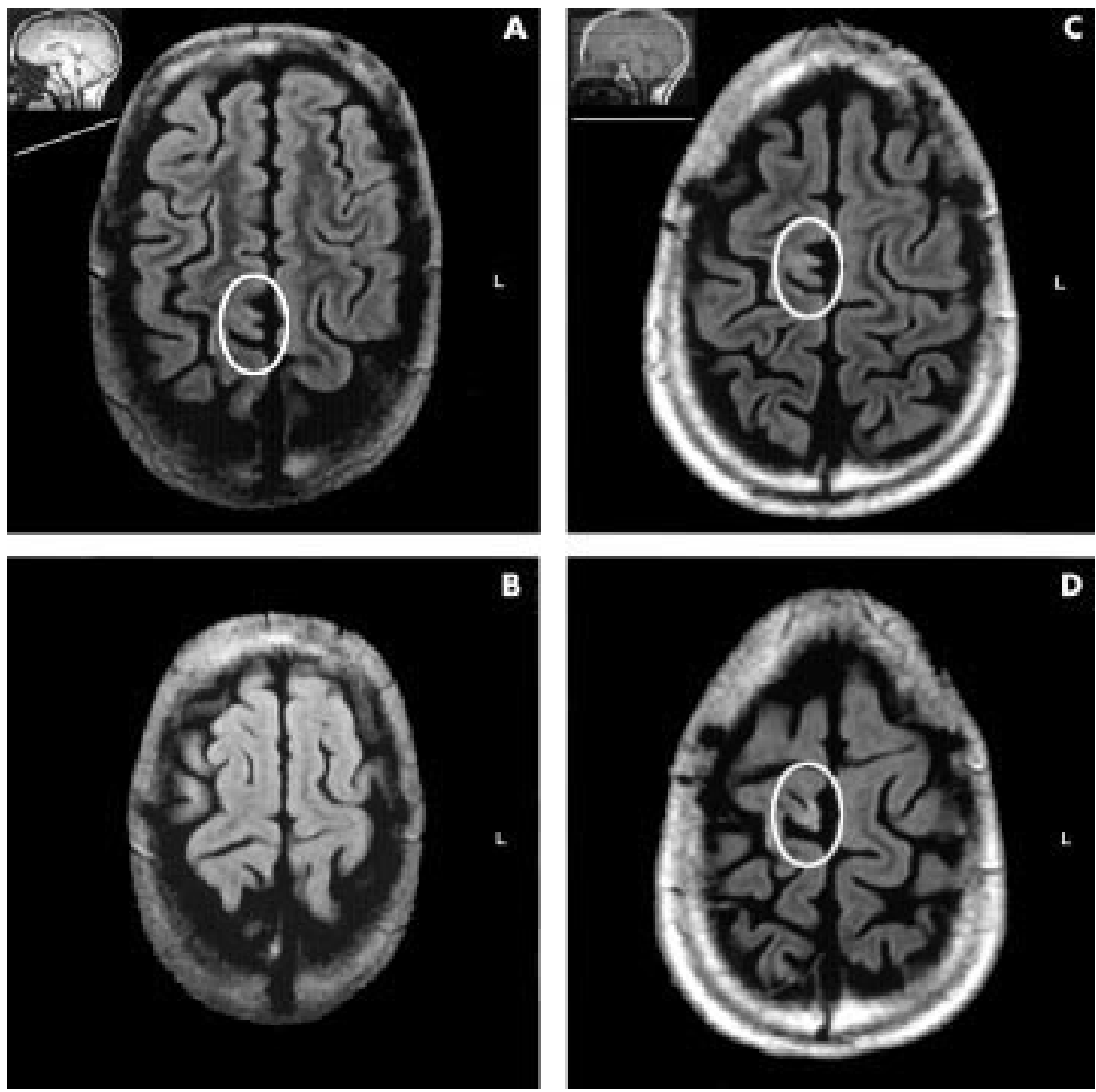

Figure 3 Superiority of a dedicated epilepsy MRI protocol: axial T2 weighted fluid attenuated inversion recovery (FLAIR) sequences of standard (A, B) and specific (C, D) MRI. Despite good image quality of standard MRI, the focal hyperintensity was only visualised in epilepsy dedicated MRI by orientation perpendicular to the sulci and gyrae that corresponds to the AC-PC plane. Scout images in the upper left corner $(\mathrm{A}, \mathrm{C}$ ) indicate different angulation (see fig 1). This example shows that even using a standardised epilepsy dedicated MRI protocol, visualisation of focal lesions may require individualisation depending on the presumed seizure onset zone. 

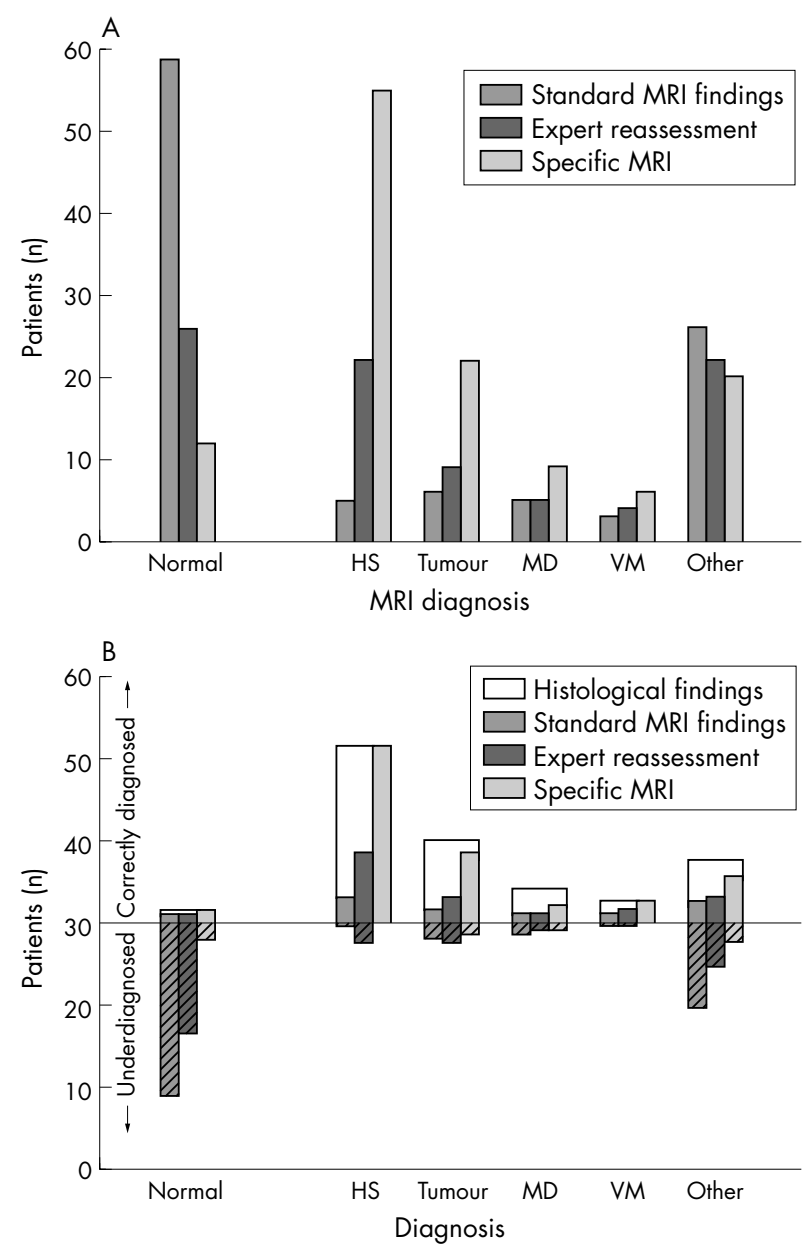

Fig 4 (A) Sensitivity for focal abnormalities of standard MRI, "expert" reassessment of standard MRI by epileptologically experienced neuroradiologists and specific MRI in 123 medically intractable epilepsy patients. Thirty four standard MRI scans classified as technically inadequate by epilepsy "experts" were included in the "normal or no focal abnormality" category. The data indicate that the sensitivity of "expert" reassessment of standard MRI is superior to standard MRI reporting by "non-experts". Epilepsy dedicated MRI is superior to all. (B) Likelihood of correct diagnosis of the three different MRI results (standard/"non-expert", standard/"expert", specific/"expert") as well as quality of MRI diagnosis in 90 operated patients. MRI misdiagnoses are shown below zero. Data indicate high specificity for epilepsy dedicated MRI and only low specificity for standard MRI as well as many misdiagnoses with standard MRI. Normal=normal or no focal abnormalities, HS=hippocampal sclerosis, $M D=$ migration disorders, $V M=$ vascular malformations, other=other focal abnormalities.

\section{Patients with known scan-scan interval $(n=90)$}

The mean interval between standard and dedicated MRI scans (scan-scan interval) was 21.6 months (range 0.6-70.7). Scanscan interval of "normal" standard MRI was 25 months (range 4.6-70.7) of "lesional" standard MRI 19 months (range: 0.6-59.4). This difference was statistically significant $(\mathrm{p}<0.001$ in all test repetitions $)$. The proportion of technically inadequate MRIs (definition see Methods section) decreased during the study period although the detection rate of "nonexperts" reporting standard MRI did not increase in the same way (table 2). The improvement of standard scan quality with time was reflected by a higher lesion detection rate when standard MRIs were reviewed by "experts".

\section{DISCUSSION}

Sensitivity for the detection of focal lesions was low if standard MRI sequences were reported by "non-experts", signifi-
Table 2 Annual sequence of standard MRI findings and "expert" reassessment of standard MRI in medically intractable epilepsy patients $(n=90)$. Quality of standard MRI examination improved during the period but external reading of MRI did not. In some patients standard MRI was classified insufficient but focal lesion could be identified anyhow

\begin{tabular}{llllll}
\hline & & \multicolumn{2}{l}{$\begin{array}{l}\text { Standard MRI } \\
\text { reading (\%) }\end{array}$} & \multicolumn{2}{l}{$\begin{array}{l}\text { "Expert" reassessment of } \\
\text { standard MRI (\%) }\end{array}$} \\
\cline { 5 - 5 } $\begin{array}{l}\text { Year of MRI } \\
\text { examination }\end{array}$ & $\mathrm{n}$ & focal lesion & & insufficient & focal lesion \\
\hline 1993 & 6 & 50 & 67 & 50 \\
1994 & 10 & 30 & 70 & 40 \\
1995 & 15 & 56 & 31 & 63 \\
1996 & 25 & 31 & 41 & 46 \\
1997 & 22 & 43 & 26 & 65 \\
1998 & 12 & 50 & 33 & 67 \\
\hline
\end{tabular}

cantly higher if the same pictures were analysed by epilepsy "experts", and higher again with dedicated epilepsy MRI protocols. In terms of likelihood of correct diagnosis, the discrepancy between standard MRI and dedicated MRI was even larger. Our data show that MRI of patients with refractory focal epilepsy should be undertaken by epileptologically experienced neuroradiologists using epilepsy specific imaging protocols.

"Non-experts" using standard MRI missed abnormalities in the mesial temporal lobe more frequently than neocortical changes. Most mistakes were made with regard to HS, the most common MRI diagnosis in medically intractable epilepsy. The discrepancy between "expert" reassessment of standard MRI and specific MRI scans of the same patients reflects the superiority of dedicated epilepsy scanning protocols in this clinical context. Such protocols include an individual orientation, thin slice thickness and high resolution. "Experts" using epilepsy dedicated MRI can detect HS with a sensitivity and a specificity of over $90 \%{ }^{11-13}$

Standard MRI was usually performed before referral for epilepsy surgery evaluation, dedicated MRI during inpatient evaluation resulting in a mean time interval between standard and dedicated MRI of almost 22 months. Patients with normal standard MRI had significantly longer scan-scan intervals, suggesting that the "normal" scan contributed to a delayed referral for presurgical evaluation. The scan-scan delay could have biased our study in favour of specific MRI because of progressive tissue changes. However, most of the detected lesions were non-progressive. Previous MRI studies of HS have shown minimal progression of hippocampal damage over time. ${ }^{14}{ }^{15}$ Patients with rapidly growing lesions, like tumours WHO grade III or IV were excluded. Tissue changes occurring during the scan-scan interval in this study are therefore unlikely to explain the different results obtained by standard and specific MRI examinations. Furthermore, "expert" reassessment of standard MRI produced markedly better results than external standard MRI reporting. Interestingly, there were no false positive findings at all in any of the non-expert acquired and reported scans in this study. The main problem detected here is therefore one of underdetection and underreporting, and not of overreporting of abnormalities. We do, however, come across false positive non-expert reports in our clinical practice and results of standard MRI should be carefully verified before a decision about epilepsy surgery is made.

Our results correspond to those of a previous study comparing standard and epilepsy dedicated MRI protocols in medically intractable temporal lobe epilepsy patients. ${ }^{10}$ In that study, standard MRI failed to show focal abnormalities in 34 of $51(67 \%)$ patients, and missed 32 of 34 temporal lesions 
including 27 cases of HS. However, the study did not include "expert" reassessment or an evaluation of scan-scan intervals. It was undertaken at a time (1993-96) when the technical differences between standard and specific MRI may have been more significant. Over the period of the present study (199699), image quality of standard MRI increased resulting in fewer inadequate scans, but the interpretation of the obtained images by "non-experts" showed no improvement. It is important to emphasise that a dedicated epilepsy MRI protocol can be performed on almost any 1.5 T MRI scanner without additional software. However, the fact that standard MRI reporting of "experts" was superior to reporting by "nonexperts" suggests that despite general improvements of image quality best results will be obtained by dedicated MRI assessed by experts at epilepsy centres. It should be noted that, since completion of this study, we have improved our dedicated epilepsy protocol further by including a coronal FLAIR sequence as well as a three dimensional Tl weighted acquisition for further postprocessing analysis such as reformatting and quantitative evaluation. We no longer acquire the sagittal Tl weighted sequence.

Patients with medically intractable epilepsy do not just have recurrent seizures. Their illness causes social stigma and disability. ${ }^{16}$ Epilepsy is a considerable social burden. ${ }^{17-19} \mathrm{~A}$ lesion detected early in the course of a seizure disorder by dedicated MRI, and good surgical prospects can have tremendous consequences for patients with medically intractable epilepsy. Quality of life improves after surgical treatment, ${ }^{20}$ early surgery is associated with better socioeconomic outcome, ${ }^{21}$ functional deficits can improve after surgical intervention, ${ }^{22}$ and the risk of injury and premature death are significantly lower after successful surgery. ${ }^{23-25}$

Epilepsy patients who do not respond to the first two antiepileptic drugs are likely to have drug resistant epilepsy. ${ }^{5}$ Our data suggest that such patients should undergo MRI using a dedicated epilepsy protocol, and that there is need for a more appropriate training of (neuro-)radiologists to read the scans by a standardised interpretation approach. ${ }^{26}$ The use of standard MRI in epilepsy should be limited to the exclusion of abnormalities, which have to be treated irrespective of epileptological considerations at the onset of the disorder like stroke or malignant brain tumours.

\section{ACKNOWLEDGEMENTS}

We would like to thank L Solymosi, J Reul, B Ostertun, and E Keller for expert (re)assessment of standard and epilepsy dedicated MRI during the period described above. Furthermore, we thank the involved epilepsy surgeons, D van Roost and J Schramm, as well as the neuropathologists, I Bluemcke and O D Wiestler, for specimens and analysis.

\section{Authors' affiliations}

J von Oertzen, S Jungbluth, M Kurthen, M Reuber, G Fernández, C E Elger, Department of Epileptology, University of Bonn, Bonn, Germany
H Urbach, Department of Radiology and Neuroradiology, University of Bonn

Competing interests: none declared.

\section{REFERENCES}

1 Annegers JF. The epidemiology of epilepsy. In: Wyllie E, ed. The treatment of epilepsy: priciples and practice. Baltimore: Williams and Wilkins, 1996:165-72.

2 Mattson RH, Cramer JA, Collins JF. A comparison of valproate with carbamazepine for the treatment of complex partial seizures and secondarily generalized tonic-clonic seizures in adults. The Department of Veterans Affairs Epilepsy Cooperative Study no 264 Group. N Engl J Med 1992;327:765-71

3 Mattson RH, Cramer JA, Collins JF. Prognosis for total control of complex partial and secondarily generalized tonic clonic seizures. Department of Veterans Affairs Epilepsy Cooperative Studies no 118 and No. 264 Group. Neurology 1996;47:68-76.

4 Semah F, Picot MC, Adam C, et al. Is the underlying cause of epilepsy a major prognostic factor for recurrence? Neurology 1998;51:1256-62.

5 Kwan P, Brodie M. Early identification of refractory epilepsy. N Engl J Med 2000;342:314-19.

6 Engel J Jr. Surgery for seizures. N Engl J Med 1996;334:647-52.

7 Wiebe S, Blume WT, Girvin JP, et al. A randomized, controlled trial of surgery for temporal-lobe epilepsy. N Engl J Med 2001;345:31 1-18.

8 Duncan JS. Imaging and epilepsy. Brain 1997;120 (pt 2):339-77.

9 Commission on Neuroimaging of the International League Against Epilepsy. Guidelines for neuroimaging evaluation of patients with uncontrolled epilepsy considered for surgery. Epilepsia 1998;39:1375-6.

10 McBride MC, Bronstein KS, Bennett $B$, et al. Failure of standard magnetic resonance imaging in patients with refractory temporal lobe epilepsy. Arch Neurol 1998:55:346-8.

11 Kuzniecky RI, Bilir E, Gilliam F, et al. Multimodality MRI in mesial temporal sclerosis: relative sensitivity and specificity. Neurology 1997;49:774-8.

12 Oppenheim C, Dormont D, Biondi A, et al. Loss of digitations of the hippocampal head on high-resolution fast spin-echo MR: a sign of mesia temporal sclerosis. Am J Neuroradiol 1998;19:457-63.

13 Cheon JE, Chang KH, Kim HD, et al. MR of hippocampal sclerosis: comparison of qualitative and quantitative assessments. Am J Neuroradiol 1998; 19:465-8.

14 Salmenpera T, Kalviainen R, Partanen K, et al. Hippocampal damage caused by seizures in temporal lobe epilepsy. Lancet 1998;351:35.

15 Van Paesschen W, Duncan JS, Stevens JM, et al. Longitudinal quantitative hippocampal magnetic resonance imaging study of adults with newly diagnosed partial seizures: one-year follow-up results. with newly diagnosed partial
Epilepsia 1998; 39:633-9.

16 Anonymous. In the shadow of epilepsy. Lancet 1997;349:1851

17 Baker GA, Brooks J, Buck D, et al. The stigma of epilepsy: a European perspective. Epilepsia 2000;41:98-104.

18 Krauss GL, Gondek S, Krumholz A, et al. "The scarlet E": the presentation of epilepsy in the English language print media. Neurology 2000;54:1894-8.

19 Morrell MJ, Pedley TA. "The scarlet E": epilepsy is still a burden. Neurology 2000;54:1882-3.

20 Vickrey BG, Hays RD, Rausch R, et al. Outcomes in 248 patients who had diagnostic evaluations for epilepsy surgery. Lancet 1995;346:1445-9.

21 Lendt $M$, Helmstaedter C, Elger CE. Pre- and postoperative socioeconomic development of 151 patients with focal epilepsies. Epilepsia 1997;38:1330-7

22 Helmstaedter C, Elger CE. Functional plasticity after left anterior temporal lobectomy: reconstitution and compensation of verbal memory functions. Epilepsia 1998:39:399-406.

23 Cockerell OC. The mortality of epilepsy. Curr Opin Neurol 996;9:93-6.

24 Lhatoo SD, Langan Y, Sander JW. Sudden unexpected death in epilepsy. Postgrad Med J 1999;75:706-9.

25 Hennessy MJ, Langan Y, Elwes RD, et al. A study of mortality after temporal lobe epilepsy surgery. Neurology 1999:53:1276-83.

26 Bronen RA, Fulbright RK, Kim JH, et al. A systematic approach for interpreting MR images of the seizure patient. Am J Roentgenol 1997; 169:241-7. 